\title{
LA UNIÓN EUROPEA DEL SIGLO XXI: UNA NUEVA PROYECCIÓN ESPACIAL DEL PROCESO DE INTEGRACIÓN DEL CONTINENTE EUROPEO; UN TERRITORIO AMPLIADO*
}

Juan Ignacio PLAZA GUTIÉRREZ

Universidad de Salamanca

\section{INTRODUCCIÓN: PRECISIONES CONCEPTUALES Y DELIMITACIÓN GEOGRÁFICA DEL PROYECTO}

Más allá de las consideraciones económicas e institucionales que definen al proceso de integración europea que arrancó en Roma en 1957, éste es, ante todo, un proyecto que gradualmente ha ido tomando cuerpo, forma, y que se ha ido ha ido haciendo más nítido en todo este tiempo. Un proyecto cuyo diseño se ha ido configurando paulatinamente a partir de una idea inicial pero, asimismo, cambiante al ritmo de las mutaciones sociales, económicas y políticas del continente a lo largo de toda la segunda mitad del del siglo XX. Y además es un proyecto con un reflejo territorial o geográfico bastante explícito que ha ido, igualmente, variando en esta media centuria hasta traducirse en una nueva conformación territorial que, de forma totalmente efectiva a partir de mayo de 2004, agrupa a un conjunto de 25 Estados miembros que muy poco después (año 2007) será de 27.

Esta dilatada andadura que ha conocido cinco ampliaciones, con ésta última acordada en Copenhague a finales de 2002 y sellada definitivamente en Atenas el 16 de abril de 2003, ha reavivado -entre otros muchos- el debate sobre el propio concepto de Europa, considerado como algo más que una simple realidad geográfica (¿reinventada "geopolíticamente” tras la progresiva redefinición de su mapa?); reflexión que, tan sólo a título de ensayo informativo y con formato editorial, ha merecido la atención de muy diversos columnistas en diferentes medios de comunicación escritos desde que se removieron las fronteras del

\footnotetext{
* Este trabajo representa una versión revisada y corregida de la conferencia pronunciada el 20 de enero de 2003 en el marco del «Master in European Studies. Módulo Europeo de la Universidad de Salamanca: "Unidad y Diversidad en Europa" » (Coord.: Cátedra Jean Monnet de Historia)
} 
viejo continente en sus márgenes centro-orientales a partir de $1990^{1}$. Y son precisamente tanto esta prolongada trayectoria como el espacio geográfico hacia el que se abre ahora el proceso de integración los que aconsejan que nos detengamos en ciertas puntualizaciones y matizaciones conceptuales y territoriales previas, necesarias y clarificadoras.

Con la incorporación de diez nuevos Estados a este proyecto de integración (que partiendo de su dominante concepción económica de Mercado Común ha evolucionado hasta un nuevo perfil más político, social y ciudadano que desde hace diez años pasó a denominarse institucionalmente "Unión Europea", UE) se ha tendido hacia una equivocada generalización geográfica que ha tomado como el todo a la parte más amplia de este proceso. Se habla coloquialmente, y como expresiones más difundidas, de la ampliación de la UE hacia el Este, de que Europa se abre hacia el Este, de los países del Este en la nueva Europa, etc., tendiendo a olvidar que, si bien es cierto que en términos de peso geográfico el "grueso" territorial (98.7\% de la superficie) -y demográfico (98.5\% de toda la población)- de la ampliación se corresponde con estos países, también forman parte de este proyecto dos microestados claramente mediterráneos como Malta y Chipre que, por otro lado, junto con Turquía, firmaron los respectivos acuerdos de asociación con la entonces CEE bastantes años antes que los países centro-orientales, al igual que sus solicitudes de adhesión.

De igual manera se ha tendido en muchas ocasiones a discutir la "europeidad" de parte de los nuevos Estados de Europa Central y Oriental que ahora se integran en la UE, reservando erróneamente tal condición a título más propio para los países más occidentales (en términos geográficos) del Viejo Continente. Afortunadamente la historia es sabia y aleccionadora, refresca la memoria y corrige las falsas verdades que si bien se han difundido han sido acertadamente desmontadas por autores y conocedores de la propia realidad geopolítica e histórica de Europa (Taibo; 1998$)^{2}$. En la consolidación y construcción política y cultural del continente europeo han sido precisamente muchos los pueblos, culturas y ciudades del centro y del este que han participado antes y de forma más intensa que algunos de la franja más occidental y meridional. Incluso el proceso de industrialización alcanzó más tempranamente a parte de estos territorios centrales y orientales que a algunas de las regiones que hoy, en el sur y oeste de

\footnotetext{
${ }^{1}$ Ahí están, por ejemplo, los artículos editados en el Diario EL PAÍS de Cebrián, J.L. (1991): «Los límites de Europa»; 9-XII-1991; pg. 13; o, con el mismo titulo, de Solé Tura, J. (1994): «Los límites de Europa»; 11-XI-1994; pg. 15-16; o de Sotelo, I. (1997): «¿Dónde acaba Europa?»; 7-XI-1997; o el de Ortega, A. (1999): «Un mapa de Europa»; 18-X-1999; pg. 6.

2 TAibo Arias, C. (1998): "Las otras Europas"; en Política y Sociedad, no 28; pp. 55-62.
} 
Europa, alcanzan protagonismo y expansión destacados y son portadoras de nuevos desarrollos.

Por todo ello en último término y desde una perspectiva estrictamente geográfica, hablar de los nuevos Estados miembros de una UE con 25 socios (y en pocos años con 27 , cuestión que es algo más que numérica, pues refuerza lo que a continuación subrayamos) es hablar de una UE que se ampía hacia distintas márgenes del continente, pues integra realidades territoriales que forman parte de grandes regiones geográficas claramente contrastadas, no de una UE que mira solamente al Este de Europa. Es ésta, realmente, la dimensión geográfica precisa y correcta que se impone.

El nuevo mapa del proceso de integración hace todavía más completa, por un lado, la última ampliación nórdica acogiendo a los Países Bálticos, que encuentran en Dinamarca, Suecia y Finlandia su escenario geográfico "natural" de relación, el cual gravita en torno a la cuenca marítima del Báltico (el mundo báltico-escandinavo como referencia geográfica común). Por otro lado, prolonga la ampliación por el centro y este del continente (la región de Europa Central que tradicionalmente han distinguido los geógrafos) que en 1995 avanzase ya con la incorporación de Austria y que ahora, integrando a Polonia, a la República Checa, a Eslovaquia y a Hungría, hace que la UE sea ya fronteriza con Rusia, con Bielorrusia y con Ucrania. Por otra parte, la periferia helénico-balcánica ya no contará sólo con el bastión de Grecia, sino que incorpora asimismo a Eslovenia, Estado que se sitúa en el umbral fronterizo entre las influencias propias de Centroeuropa y el mundo mediterráneo; la región y península helénicobalcánica, pues, es el tercer conjunto geográfico hacia el que se proyecta la nueva UE (y por donde ha trazado ya sus perspectivas más próximas de continuación, con la adhesión de Rumanía y Bulgaria en el año 2007 -que desde una perspectiva geopolítica y en un contexto anterior ya diluido, estaban asociados al antiguo concepto del "Este"-). Una nueva Unión que, finalmente, se amplía hacia el centro y este de la cuenca mediterránea, hacia la Europa del sur o meridional, acogiendo a microestados como Malta o Chipre.

\section{II: ¿QUÉ REPRESENTA LA AMPLIACIÓN DE LA UNIÓN EUROPEA EN LOS INICIOS DEL SIGLO XXI?. ALGUNOS INDICADORES COMPARATIVOS.}

La quinta ampliación de la UE, proyectada geográficamente sobre los territorios antes reseñados, encuentra su punto de partida y precedente último en la remoción de fronteras, estados e instituciones de la antigua Europa del Este que se produjo a partir de 1990. Las extintas democracias populares situadas en la órbita soviética orientaron con marcada ansiedad su mirada económica y de seguridad hacia occidente: la UE y la OTAN se convirtieron, así, en referentes 
imprescindibles para estos Estados. A partir de esta nueva realidad geopolítica continental que comenzó a dibujarse tras la caída del muro de Berlín, la desaparición de la Unión Soviética y la reunificación de Alemania, el proceso de ampliación comenzó a adquirir progresivamente más cuerpo y tomó un ritmo que de forma acelerada terminó por definirse a finales del año 2002 en la cumbre de Copenhague.

Cuadro 1. Cronología de la quinta ampliación de la UE

\begin{tabular}{lllll}
\hline \multicolumn{1}{c}{ País } & $\begin{array}{c}\text { Fecha de la firma } \\
\text { del acuerdo de aso- } \\
\text { ciación }\end{array}$ & $\begin{array}{c}\text { Fecha de en- } \\
\text { trada en vi- } \\
\text { gor del } \\
\text { acuerdo }\end{array}$ & $\begin{array}{c}\text { Fecha de la soli- } \\
\text { citud oficial de } \\
\text { adhesión }\end{array}$ & $\begin{array}{c}\text { Fecha prevista } \\
\text { para la adhesión }\end{array}$ \\
\hline Bulgaria & Marzo de 1993 & Febrero 1995 & Diciembre 1995 & 2007 \\
Rep. Checa & Octubre de 1993 & Febrero 1995 & Junio de 1996 & 2004 \\
Chipre & Diciembre de 1972 & Junio 1973 & 3 julio de 1990 & 2004 \\
Eslovaquia & Octubre de 1993 & Febrero 1995 & Junio de 1995 & 2004 \\
Eslovenia & Junio de 1996 & Febrero 1999 & Junio de 1996 & 2004 \\
Estonia & Junio de 1995 & --- & Noviembre 1995 & 2004 \\
Hungría & Diciembre de 1991 & Febrero 1994 & Marzo de 1994 & 2004 \\
Letonia & Junio de 1995 & --- & Octubre de 1995 & 2004 \\
Lituania & Junio de 1995 & --- & Diciembre 1995 & 2004 \\
Malta & Diciembre de 1970 & Abril de 1971 & 16 julio de 1990 & 2004 \\
Polonia & Diciembre de 1991 & Febrero 1994 & Abril de 1994 & 2004 \\
Rumanía & Febrero de 1993 & Febrero 1995 & Junio de 1995 & 2007 \\
Turquía & Septiembre de 1963 & Dicbre. 1964 & 14 abril de 1967 & i. \\
\hline
\end{tabular}

Sin embargo, tal y como antes apuntamos, países como Malta y Chipre ya habían iniciado con anterioridad este proceso (el primero firmó su acuerdo de asociación en diciembre de 1970 y el segundo dos años después; ambos solicitaron oficialmente su adhesión en julio de 1990), aunque en el caso chipriota su fracturación y división constituyeron un obstáculo de primer orden, no resuelto definitivamente todavía hoy pero sí bastante más encauzado. En último término, la generalización de las sucesivas firmas de los acuerdos de asociación, así como de la solicitud oficial de adhesión de los países de Europa Central y Oriental en la década de los noventa (CUADRO 1), englobó tanto a Malta y Chipre como a los países bálticos, a los de Europa Central y a algunos de los balcánicos en una estrategia de pre-adhesión y de camino hacia la futura integración que culminó en diciembre de 2002. Acordada definitivamente una ampliación de la UE a diez miembros a partir del año 2004 y a otros dos más (Rumanía y Bulgaria) desde el 2007, ¿qué representa, pues, ésta?.

Siguiendo las estadísticas comunitarias proporcionadas tanto por el Segundo Informe sobre la Cohesión Económica y Social como por la Estrategia Territorial Europea (ETE), desde el punto de vista demográfico una próxima UE a 25 
supondrá un crecimiento de un $20,5 \%$ de sus efectivos (con una UE a 27 en el año 2007 sería del 28\%) y territorialmente de un 23\% (UE-27: 34\%). Los diez nuevos miembros, así, tendrían un peso poblacional en la próxima UE del 17\% y una representatividad territorial del $18,6 \%$, cifras que se elevarían, respectivamente, al $25 \%$ y $25,5 \%$ si hablamos de doce nuevos socios (a partir del 2007); esto es, representarían una cuarta parte tanto de la población como del territorio de la UE.

Ahondando algo más en estas consideraciones, y siguiendo los certeros análisis ofrecidos por las fuentes arriba señaladas, son especialmente significativas algunas características que con la ampliación a 10 y 12 nuevos Estados tomará tanto la UE, de forma general, como, más particularmente, este conjunto geográfico de nuevos miembros (CUADRO 2 y CUADRO 3). Por ejemplo, sólo Rumanía y Polonia serán "países grandes" en comparación con los otros nuevos países. De hecho, frente a ellos, los tres países bálticos, Eslovenia y los dos Estados insulares mediterráneos (Chipre y Malta) aportan a la nueva UE un incremento del grupo de "países pequeños" (de menos de cuatro millones de habitantes), formado hasta entonces sólo por Luxemburgo e Irlanda.

También la densidad media de población de los países de la ampliación arroja valores algo inferiores a los de la UE-15 (101,8 hab./ $/ \mathrm{km}^{2}$ ó 97,4 según hablemos, respectivamente, de 10 ó 12 nuevos miembros, frente a 115). Ahora bien, analizando las diferencias internas de densidad en uno u otro conjunto de los referidos (UE-15 y grupo de nuevos países) los resultados se invierten: por Estados son mucho más acusados los contrastes dentro de la UE-15 (la densidad media más elevada -Países Bajos- es 24,5 veces mayor que la de Finlandia -la más baja-) que en el grupo de 10 países que próximamente se incorporarán (la de la República Checa sólo es 4,1 veces mayor que la de Estonia). Incluso la densidad de población de los nuevos Estados miembros menos poblados (los Países Bálticos) es superior a la de los Estados nórdico-escandinavos de la UE15 (Finlandia y Suecia).

Y también la distribución territorial de la población de la próxima UE ampliada marcará modelos distintos entre la UE-15 y los 10 países que se adherirán oficialmente en el año 2004. En éstos es una distribución geográficamente más concentrada que en los que forman la UE-15, pues mientras el $62 \%$ de los habitantes de los 10 países viven en regiones fronterizas (lo que que hace que la ampliación represente un reforzamiento de la cooperación transfronteriza entre ellos y la UE-15, verdadero gran reto para la futura política europea de desarrollo territorial), en la UE-15 sólo lo hace el 15\%.

Pero también se marcarían los contrastes socioeconómicos en la próxima UE a 25 (efectiva desde el próximo 1-V-2004), pues los diez nuevos países tendrían 
una renta media del $44 \%$ comparada con la de la actual UE a 15 y su PIB global (en el año 2001), que era de 404.000 millones de euros, representaba sólo el 5\% del PIB total de la Europa comunitaria de quince miembros (que ascendía en el año 2001 a 8,8 billones de euros). De hecho, la región más rica de ésta, Hamburgo (con un PIB/hab. que es el 185,5\% de la media de la UE), lo es en una proporción 6,7 veces superior a la región más pobre de los próximos socios (la región de Podkarpackie, en Polonia, cuyo PIB/hab. es el 27,4\% de la media de la UE a 15). Por Estados, el PIB/hab. más elevado de la UE-15 (el de Luxemburgo) es 7,1 veces superior al más bajo de los 10 nuevos Estados (el de Letonia); e igualmente comparando el peso mundial de la producción bruta total de ambos conjuntos el distanciamiento es evidente: la UE-15 concentra casi una quinta parte el total mundial (el 19,94\% del PIB total del mundo) mientras que los 10 países del centro-este y sur de Europa tan sólo detentan el 1,79\%.

Así pues, la ampliación se traducirá en un aumento de la población eurocomunitaria, en un incremento territorial de la UE y en un reforzamiento - $¿ y$ crecimiento?- de la brecha socioeconómica que separará a los ciudadanos más ricos de los más pobres. Esta última consecuencia es la que ha hecho que también la ampliación haya vuelto a reabrir el debate de la solidaridad interregional dentro de la Unión, la reforma de la futura política regional europea y la reasignación y redistribución de los fondos estructurales, pues esta apertura a nuevos socios, como sucedió en anteriores ocasiones, ha representado, igualmente, la incorporación de nuevos problemas y dinámicas claramente alejados de los niveles de expansión y de desarrollos emergentes que conocen las regiones mediterráneas, noroccidentales y perialpinas.

\section{LOS NUEVOS TERRITORIOS DE LA AMPLIACIÓN. EL PESO DESTACADO DE LOS PAÍSES DE EUROPA CENTRAL Y ORIENTAL.}

Tal y como en su momento recordamos, a excepción de Chipre y Malta, dos microestados insulares en el Mediterráneo que en términos territoriales y demográficos no suponen ni tan siquiera el 2\% del nuevo grupo de países miembros de la UE, la ampliación del año 2004 se extiende mayoritariamente por tierras de Europa central y oriental, desde su interior (polacos, checos, eslovacos y húngaros) hasta la periferia báltica (por el norte) y los bordes balcánicos (por el sur).

Todo este territorio centro-oriental del continente ha adquirido, ante todo, un protagonismo destacado. Un protagonismo histórico por cuanto a lo largo y ancho del mismo, en el proceso de evolución y configuración geohistóricas de Europa, se han sucedido constantes vaivenes de pueblos, de culturas y de fronteras (desde las grandes rutas del comercio en épocas pretéritas hasta los grandes 
movimientos y migraciones que indistintamente han atravesado todas estas regiones). En palabras de Sellier (1995) ${ }^{3}$, ha sido éste un "espacio cerrado", aprisionado por la geografía política que ha hecho que los confines de distintos imperios (alemán, ruso, austro-húngaro, otomano) le festoneasen por todos sus costados. Protagonismo histórico también por la inestabilidad territorial que, en mucha mayor medida que en otros bordes del continente, ha caracterizado a estos espacios antes, durante y después del siglo XX (aún a partir de 1989 y en los años siguientes se siguió reproduciendo este proceso): paísea que aparecen, desaparecen y reaparecen con distintos trazados, otros que varían y se separan, etc. Y un protagonismo más reciente (último decenio del siglo XX) por su progresivo acercamiento y vinculación con la Europa comunitaria, porque con ello este territorio ha contribuido a ir perfilando con más nitidez la denominada "nueva Europa". Y en ello han actuado motivos -recapitulando ideas antes ya desgranadas y de forma muy genérica- que tienen que ver con esa progresiva tendencia que, en mayor o menor medida, ha hecho que todos estos Estados encontrasen en la actual UE y en el bloque geopolítico y geoeconómico "occidental" en que se enmarca sus nuevas referencias económicas y de seguridad.

La delimitación, el concepto y el criterio de lo que es actualmente todo este territorio tampoco concitan un consenso científico y geopolítico unánime. Cierto es que se trata de un "espacio de transiciones" generalizadas en lo político (progresiva definición de instituciones y sistemas políticos democráticos que han avanzado de modo muy desigual en unos y otros países) y en lo económico (hacia una economía de mercado). Hay autores (AHIJADO; 1999) ${ }^{4}$ que, dada la centralidad que en el proceso de definición de una nueva Europa están consiguiendo estos países que próximamente se incorporarán a la UE, catalogan a este territorio como "una tercera banana europea", en contraposición a las otras dos que se extienden por el noroeste continental y por las periferias alpinas y mediterráneas. Es un espacio en recomposición, pero ello no quiere decir que sea, ni mucho menos, un espacio homogéneo; ni tan siquiera lo fue cuando todos estos países estaban bajo la influencia de un mismo sistema políticoeconómico como el de economía estatalizada y planificación centralizada, sistema tipo soviético, no fue entonces tampoco un espacio coherentemente estructurado. Es un itsmo entre el huso Mar del Norte-Mar Adriático por el borde oeste y el huso Mar Báltico-Mar Negro por el este.

\footnotetext{
${ }^{3}$ Sellier, A. y Sellier, J. (1995): Atlas de los pueblos de Europa Central y Oriental; Acento Editorial; Madrid.

${ }^{4}$ AhiJADO, M. y OsunA, R. (1999): Unión Económica y Monetaria Europea. La ampliación al Este I. Teoría de la transición, hechos estilizados y el punto de vista comunitario; Ed. Pirámide; Madrid.
} 
Cuadro 2. Indicadores socioeconómicos comparados UE-15 / países candidatos

\begin{tabular}{|c|c|c|c|c|c|}
\hline País & $\begin{array}{l}\text { PIB/hab. } \\
\text { (\$ en } \\
\text { PPA) (año } \\
2000)\end{array}$ & $\begin{array}{c}\text { Lugar que } \\
\text { ocupa en el } \\
\text { mundo según } \\
\text { PIB/Hab. }\end{array}$ & $\begin{array}{l}\% \text { en el PIB } \\
\text { total mundial }\end{array}$ & $\begin{array}{l}\text { IDH (Indica- } \\
\text { dor Desarro- } \\
\text { llo Humano) } \\
\text { (año 2000) }\end{array}$ & $\begin{array}{l}\text { Lugar que } \\
\text { ocupa en el } \\
\text { mundo se- } \\
\text { gún IDH }\end{array}$ \\
\hline \multicolumn{6}{|c|}{ UNIÓN EUROPEA A 15 Y LOS 10 PAÍSES CANDIDATOS DEL AÑO 2004} \\
\hline Luxemburgo & 50.061 & 1 & 0,04 & 0,925 & 16 \\
\hline Irlanda & 29.866 & 6 & 0,24 & 0,925 & 18 \\
\hline Dinamarca & 27.627 & 10 & 0,34 & 0,926 & 14 \\
\hline Bélgica & 27.178 & 11 & 0,61 & 0,939 , & 4 \\
\hline Austria & 26.765 & 12 & 0,47 & 0,926 & 15 \\
\hline Países Bajos & 25.657 & 17 & 0,91 & 0,935 & 8 \\
\hline Alemania & 25.103 & 19 & 4,58 & 0,925 & 17 \\
\hline Finlandia & 24.996 & 20 & 0,29 & 0,930 & 10 \\
\hline Suecia & 24.277 & 22 & 0,49 & 0,941 & 2 \\
\hline Francia & 24.223 & 23 & 3,22 & 0,928 & 12 \\
\hline Italia & 23.626 & 24 & 3,11 & 0,913 & 20 \\
\hline Reino Unido & 23.509 & 25 & 3,13 & 0,928 & 13 \\
\hline Chipre & 20.824 & 28 & 0,03 & 0,883 & 26 \\
\hline España & 19.472 & 32 & 1,75 & 0,913 & 21 \\
\hline Eslovenia & 17.367 & 37 & 0,08 & 0,879 & 29 \\
\hline Portugal & 17.290 & 38 & 0,38 & 0,880 & 28 \\
\hline Malta & 17.273 & 39 & 0,01 & 0,875 & 30 \\
\hline Grecia & 16.501 & 42 & 0,38 & 0,885 & 24 \\
\hline Rep. Checa & 13.991 & 46 & 0,32 & 0,849 & 33 \\
\hline Hungría & 12.416 & 48 & 0,27 & 0,835 & 35 \\
\hline Eslovaquia & 11.243 & 51 & 0,13 & 0,835 & 36 \\
\hline Estonia & 10.066 & 54 & 0,03 & 0,826 & 42 \\
\hline Polonia & 9.051 & 59 & 0,82 & 0,833 & 37 \\
\hline Lituania & 7.106 & 74 & 0,06 & 0,808 & 49 \\
\hline Letonia & 7.045 & 76 & 0,04 & 0,800 & 53 \\
\hline \multicolumn{6}{|c|}{ PAÍSES CANDIDATOS PARA EL 2007 Y TURQUÍA } \\
\hline Turquía & 6.974 & 77 & 0,99 & 0,742 & 85 \\
\hline Rumanía & 6.423 & 79 & 0,32 & 0,775 & 63 \\
\hline Bulgaria & 5.710 & 89 & 0,10 & 0,779 & 62 \\
\hline
\end{tabular}

FUENTE: El Estado del Mundo. Anuario económico geopolítico mundial 2003; Akal 
Cuadro 3. Capitales, peso territorial y ocupación humana del espacio de una Unión Europea ampliada

\begin{tabular}{|c|c|c|c|c|}
\hline País & Capitales & $\begin{array}{c}\text { Superficie } \\
\left(\mathrm{Km}^{2}\right)\end{array}$ & Población & Densidad \\
\hline \multicolumn{5}{|c|}{ UNIÓN EUROPEA A 15 Y LOS 10 PAÍSES CANDIDATOS DEL AÑO 2004} \\
\hline Luxemburgo & Luxemburgo & 2.586 & 442.000 & 171,0 \\
\hline Irlanda & Dublín & 70.820 & 3.841 .000 & 55,8 \\
\hline Dinamarca & Copenhague & 43.070 & 5.333 .000 & 125,7 \\
\hline Bélgica & Bruselas & 30.500 & 10.264 .000 & 312,7 \\
\hline Austria & Viena & 83.850 & 8.075 .000 & 97,6 \\
\hline Países Bajos & Amsterdam & 34.000 & 15.930 .000 & 470,2 \\
\hline Alemania & Berlín & 357.050 & 82.007 .000 & 229,9 \\
\hline Finlandia & Helsinki & 337.010 & 5.178 .000 & 17,0 \\
\hline Suecia & Estocolmo & 449.960 & 8.833 .000 & 21,5 \\
\hline Francia & París & 547.026 & 59.453 .000 & 108,1 \\
\hline Italia & Roma & 301.225 & 57.503 .000 & 195,5 \\
\hline Reino Unido & Londres & 244.046 & 59.452 .000 & 247,2 \\
\hline España & Madrid & 504.782 & 39.921 .000 & 79,9 \\
\hline Portugal & Lisboa & 92.080 & 10.033 .000 & 109,7 \\
\hline Grecia & Atenas & 131.944 & 10.623 .000 & 82,4 \\
\hline Chipre & Nicosia & 9.250 & 778.000 & 84 \\
\hline Malta & La Valetta & 320 & 386.000 & $1.206,0$ \\
\hline Rep. Checa & Praga & 78.370 & 10.262 .000 & 133,0 \\
\hline Eslovaquia & Bratislava & 49.500 & 5.382 .000 & 112,0 \\
\hline Hungría & Budapest & 93.030 & 10.076 .000 & 109,0 \\
\hline Eslovenia & Ljublijana & 20.250 & 1.989 .000 & 99,0 \\
\hline Polonia & Varsovia & 312.680 & 38.740 .000 & 127,0 \\
\hline Estonia & Tallin & 45.225 & 1.412 .000 & 33,0 \\
\hline Lituania & Vilnius & 65.200 & 3.682 .000 & 57,0 \\
\hline Letonia & Riga & 64.500 & 2.389 .000 & 39,0 \\
\hline \multicolumn{5}{|c|}{ PAÍSES CANDIDATOS PARA EL 2007 Y TURQUÍA } \\
\hline Rumanía & Bucarest & 237.500 & 22.402 .000 & 97,0 \\
\hline Bulgaria & Sofía & 110.910 & 8.279 .000 & 75,0 \\
\hline Turquía & Ankara & 779.450 & 65.546 .000 & 85,0 \\
\hline
\end{tabular}


La geografía y la geopolítica han aportado, desde hace ya mucho tiempo, una gran variedad de denominaciones con las que intentar dar concepto y delimitación a este territorio. Pero ni dentro de la misma ciencia geográfica hay acuerdo cuando se utiliza uno mismo de estos conceptos, tal es el caso del de "Europa Central", cuya proyección espacial y regional no representan lo mismo, por ejemplo, para dos geógrafos de una misma época como De Martonne y Ancel. En los años treinta del siglo XX ambos tenían representaciones distintas para todo este conjunto ${ }^{5}$.

Sin embargo, sí parece compartirse la afirmación de que el antiguo concepto de "Europa del Este" o "Países del Este", vigente de los años cincuenta a principios de los noventa del siglo XX, impuesto desde una perspectiva geopolítica occidental (TAIBO; 1995) ${ }^{6}$ que otorgaba una categoría política, social y económica a estos Estados (cordón defensivo y de sguridad, glacis estratégico, por parte de la antigua URSS frente a Occidente), no es representativo de un territorio cuyo sentido geográfico recupera la consolidada denominación, más específica, de "Europa central y oriental". Y así se incorpora a la terminología institucional que utiliza la misma UE cuando habla de la ampliación: Países de Europa Central y Oriental (PECO). De igual modo surgieron intentos de revitalización de otro viejo concepto como el de "Mitteleuropa", basado en raíces culturales y nacionales y difundido en el siglo XIX pero, posteriormente, burda y groseramente sesgado por la deformación que de él hizo el nazismo y los afanes expansionistas desmesurados. Algunos representantes del mundo intelectual más crítico pretendieron desempolvar su uso y significado a finales de la década de los años ochenta, poco antes de la sucesión de acontecimientos que transformaron a finales del siglo XX todo este territorio, pero no lo consiguieron dado que las preocupaciones, intereses y orientaciones de las sociedades de estos países apuntaban en distinta dirección.

Los franceses (Foucher; Rey; etc.), por su parte, han contribuido a incrementar esta diversidad con la incorporación y acuñación de otros conceptos ciertamente expresivos. Tal es el caso de lo que ellos denominan "Europe médiane" y "Europe entre deux", significativos términos que subrayan el papel que ejerce la situación geográfica de todo este conjunto dentro del viejo continente y desde

\footnotetext{
${ }^{5}$ De Martonne (1930), hablaba de Europa Central (donde incluía a Alemania, Suiza, Polonia, Checoslovaquia, Austria, Hungría y Rumanía) y de la Península mediteránea de los Balcanes. Ancel (1937), por su parte, hablaba de una "nueva Europa Central" coincidente con la Europa danubiana -término geográfico utilizado para designar un conjunto geopolítico que él llamó "Habsburguia"- y que agrupaba a los nuevos Estados formados en 1919-1920 procedentes de la antigua Austria-Hungría (el "imperio bicéfalo"): Austria, Checoslovaquia, Hungría, Rumanía y Yugoslavia.

${ }^{6}$ TAibo Arias, C. (1995): Crisis y cambio en la Europa del Este; Alianza Editorial; Madrid.
} 
una perspectiva no exclusivamente "locacional" sino también dinámica. A todos estos conceptos muy sucintamente desgranados qué duda cabe que deben añadirse, igualmente, los que la propia geografía regional tradicional del continente europeo ha ido creando. Es así como todo este territorio que en breve configurará una nueva macrorregión periférica de la nueva UE se distribuye por áreas diferenciadas que incluyen a la "Europa balcánica", la "Europa del sureste", la "Europa bajodanubiana o países danubianos", etc.

Es, igualmente, todo este conjunto una realidad geográfica plural y compleja, marcada por una acusada diversidad interna. Característica ésta que hunde sus raíces en una acendrada evolución histórica y social donde se entrecruzan las fragmentaciones religiosas de este espacio (áreas del cristianismo latino, católico y protestante de Europa central frente a las regiones y sociedades de Europa oriental estructuradas por la ortodoxia y el islam), con las geopolíticas (cuatro imperios que cabalgaron sobre estos territorios). Igualmente esta pluralidad se refuerza con la constitución de los Estados-Nación después de la primera guerra mundial y las consecuencias que fueron derivándose de este proceso: ruptura y fragmentación de muchas naciones por nuevas delimitaciones, separaciones y creación de minorías en otros territorios, subsistencia de viejos límites conflictivos, diversidad de pueblos que comienzan un proceso de reautoafirmación, etc.

Es además, en último término, un amplio conjunto cuya individualización y singularidad más recientes responden al intenso proceso de transformaciones $y$ cambios que se han desarrollado en todos estos Estados ${ }^{7}$, así como en la aparición de problemas diferenciados y en las distintas tendencias de evolución que se han ido definiendo, todo ello en dimensiones muy variadas: en lo político, en lo social, en lo económico y en lo propiamente territorial. La redefinición política e institucional es quizá uno de los resultados más evidentes de estos procesos de cambio que se han extendido en tan sólo trece años desde el Báltico a las riberas del Adriático y del Mar Negro; un resultado, en definitiva, estrechamente vinculado a la transición política (hacia sistemas democráticos efectivos y plurales, más abiertos) como manifestación más expresiva de la geopolítica europea de finales del siglo XX. En este contexto se enmarcan acciones muy diversas: distintos modelos de transición política (más evolucionados, más retar-

\footnotetext{
${ }^{7}$ Sólo a título de selección, pueden señalarse dos obras que constituyen muy buenos referentes para un análisis más detallado sobre todos estos procesos de cambio desarrollados en esta región en el último decenio del siglo XX. Por un lado, la obra de LAVIGNE, M. (1995): Del socialismo al mercado, Ed. Encuentro, Madrid, que aborda la transición económica de la antigua Europa del Este. Por otro, el trabajo colectivo coordinado por FLORES, G. Y LUENGO, F. (2000) sobre el balance de una década de transformaciones en los países del Centro y Este de Europa: Tras el muro: diez años después de 1989; Ed. El Viejo Topo, Barcelona.
} 
dados), una generalizada redefinición propiamente política e institucional (nuevas constituciones con que se van dotando cada uno de los Estados, configuración de nuevos Estados y desaparición de otros anteriores, trazado de nuevas fronteras, establecimiento de nuevas vinculaciones y relaciones internacionales -con la consiguiente recomposición de "ejes" y de áreas regionales-, etc.) ${ }^{8}$.

De igual manera, los cambios que afectan a toda la región se traducen en otras manifestaciones que han cobrado un protagonismo excepcional: se transforman los flujos migratorios, que empujan a miles y miles de ciudadanos de la antigua Europa del Este hacia los países de la Unión Europea en busca de mejores condiciones de vida y en busca de oportunidades; se empiezan a querer definir nuevos esquemas de articulación a partir de nuevos centros urbanos (Berlín, Praga, Varsovia y Budapest) y ejes de comunicaciones que refuerzan la perspectiva de una Europa central con cada vez más proyección funcional; cambios en los modelos de organización territorial: los procesos de descentralización avanzan desigualmente en los nuevos Estados, apoyándose además en la configuración de nuevos estatutos políticos que les definen (en todo ello tiene parte de responsabilidad, asimismo, el papel que han desempeñado los Fondos Estructurales y la futura Política Regional Europea, contexto al que se adscriben los nuevos países candidatos a la adhesión en la UE, pues ha movido a la creación de nuevas estructuras regionales de distribución de poder y competencias como configuración de una infraestructura político-administrativa que propicie la ejecución de instrumentos y acciones vinculados a la cohesión económica,

\footnotetext{
${ }^{8}$ Siquiera sea a título de ejemplo, no está de más que recordemos algunos de los hechos ya más concretos en que se han traducido todas estas manifestaciones de cambio. Desaparece la antigua Yugoslavia con la aparición, en su lugar de cinco nuevos Estados entre 1990 y 1995; incluso la más reciente evolución del "estado heredero" de la ex-Yugoslavia (Serbia y Montenegro) aprobó en febrero de 2003 una nueva denominación (Unión de Serbia y Montenegro) y hasta puso "fecha de caducidad" a su relación, proponiendo una revisión de la misma para el año 2006. Pero además las transformaciones geopolíticas de la antigua Yugoslavia trajeron consigo enquistamientos de problemas específicos (Kosovo, Macedonia) y soluciones de muy escasa consistencia cuya evolución aún es incierta (el reparto de Bosnia-Herzegovina). También desapareció un viejo Estado federal y en su lugar surgen la República Checa y Eslovaquia; se produce la reunificación de Alemania (extinguiéndose la separación entre la antigua RDA y la antigua RFA); e igual sucede con la que era Unión Soviética, sustituida por la Comunidad de Estados Independientes en la que no se integran los Países Bálticos, que logran su independencia en 1991 y evolucionan al margen de esta nueva figura. Y se asiste a un intento de "reunificación moldava"; y surge el problema del enclave de Königsberg; y estallan los conflictos en el Cáucaso, etc. En relación con la aprobación de nuevas Constituciones ha de decirse, asimismo, que en el escaso plazo de tiempo de su nueva configuración como Estados desligados de antiguas formas desparecidas ya, muchos de ellos han procedido además a efectuar varias reformas de sus respectivas nuevas Cartas Magnas: Croacia se dotó de una nueva en diciembre de 1990 y ha efectuado ya seis reformas de ella; Letonia restauró en agosto de 1991 su vieja constitución de 1922 y ha efectuado ya tres reformas; la de Hungría (de 1949) ha sido reiteradamente enmendada en seis ocasiones.
} 
social y territorial). Y junto a estas manifestaciones están presentes otras como la reconstrucción urbana, la recomposición del tejido espacial productivo (reconversión), o la regeneración paisajística y ambiental abordada como necesidad frente al deterioro ocasionado por una localización y política industrial desorientadas, obsoletas y contaminantes.

\section{UNA PARTE DEL TERRITORIO EUROPEO SE REENCUENTRA Y RESITÚA EN EL VIEJO CONTINENTE}

En último lugar, con la ampliación de la UE hacia los nuevos Estados de Europa central y oriental (también a Chipre y a Malta), puede afirmarse que una parte del territorio continental se reencuentra con su contexto social y político y vuelve a situarse en el esquema geopolítico y de relaciones internacionales de Europa. Por un lado se insertan en nuevos escenarios internacionales de integración (nuevas instituciones geopolíticas y geoestratégicas) de escala continental (la OSCE, el Consejo de Europa, la OTAN), a las que se van incorporando antes unos que otros y con convicciones desiguales en sus respectivas sociedades. El Consejo de Europa, concretamente, se convierte en la antesala de recepción de estos nuevos Estados en su tránsito hacia la recuperación y normalización de su presencia institucional. Pero al mismo tiempo esta integración se produce también en escenarios de dimensión más regional que constituyen espacios de relación natural para unos y otros de estos países: el Consejo de Estados del Mar Báltico (CBSS), la Cooperación Económica del Mar Negro (CEMN / BESC), la Iniciativa Centro Europea (ICE / CEI), el Pacto de Estabilidad del Sureste Europeo (PSESE), o el acuerdo de libre comercio centroeuropeo (CEFTA $)^{9}$.

Por otra parte, su reencuentro con el viejo continente se ve facilitado por la aparición de nuevas estructuras de cooperación y desarrollo que adquieren un reflejo territorial evidente. Espacios, actividades y programas de cooperación a escala continental constituyen las nuevas categorías y figuras mediante las que los países de la ampliación se resitúan en Europa. Así, las funciones y actividades centradas en los principales núcleos urbanos y regiones de estos territorios constituyen una primera vía para tal cometido: la cooperación transnacional y transfronteriza; el desarrollo territorial abordado de forma integrada; o la tipificación de las llamadas "ciudades-puerta", son ejemplos de estas nuevas dinámicas cuya precisión y definición se encuentran recogidas en los documentos-base

\footnotetext{
${ }^{9}$ Las siglas utilizadas (a excepción de CEMN e ICE) se corresponden con su denominación oficial en lengua inglesa. Así el CBSS equivale al Council of the Baltic Sea States; la BESC a la Black Sea Economic Cooperation; CEI a Central European Iniciative; PSESE al Stability Pact for South Eastern Europe y CEFTA al Central European Free Trade Agreement.
} 
sobre los que se ha trazado la nueva estructura de configuración territorial de Europa y los fundamentos de su desarrollo y ordenación: la Estrategia Territorial Europea (ETE) y los Principios para el Desarrollo Territorial Sostenible del continente europeo.

De igual manera los programas y los espacios de cooperación proyectados desde la UE alcanzan a todos estos territorios de forma específica. Fue el caso de la segunda fase de la iniciativa comunitaria INTERREG C (INTERREG II C, 1994-1999), donde se definieron como ámbitos espaciales de cooperación la región del Báltico; el área Adriático-Danubio-Europa Central y Sudoriental (el llamado territorio CADSES, siglas derivadas de su denominación en inglés); el Mediteráneo suroriental (ARCHIMED); o el Espacio alpino/Alpes orientales. Asimismo, el INTERREG III B (2000-2006) mantiene estos ámbitos e incorpora otros nuevos (Mediterráneo occidental-Alpes latinos). Y todo ello se refleja en directrices territoriales adaptadas a cada caso, las denominadas "Visiones"" como la VASAB 2010 (Vision and Strategies Around the Baltic Sea: Visión espacial para la región del Mar Báltico 2010: del modelo a la acción) o la VISION PLANET (Estrategias para una ordenación territorial integrada en el espacio CADSES).

A todo ello se suman también las "euroregiones", de carácter transfronterizo y que se encuentran muy desarrolladas en algunas regiones de Alemania, la República Checa y Polonia (Pomerania; Viadrina; Spree/Boder; Neisse; Elba/Labe; Erzgebirge Central; Egrensis), al igual que las redes e infraestructuras de relación territorial a gran escala continental (TINA: Transport Infrastructure Needs Assessment). Aquí han de mencionarse la propia Red Transeuropea de Transporte, los Corredores Transeuropeos de Transporte y las Zonas Paneuropeas de Transporte (éstas últimas son la del Mar de Barentz/Mar Euro-Ártico; la Cuenca del Mar Negro; la Cuenca Mediterránea; y la zona Mar Adriático/Mar Jónico), verdaderas piezas fundamentales para la articulación e integración de estos territorios centro-europeos en el viejo continente y que actúan como condiciones básicas para promover su dinamismo y favorecer los intercambios.

Se completa, finalmente, todo este reencuentro de Europa Central y Oriental en/con el continente mediante los instrumentos financieros y de ayuda/cooperación de origen eurocomunitario: programas y medios económicofinancieros específicos diseñados desde la UE en dirección a estos países de la ampliación. La futura Política Regional Europea (PRE), íntimamente relacionada con la Estrategia Territorial Europea (ETE), se convierte en la piedra de toque de todas estas relaciones. La segunda parte de la década de los años noven-

${ }^{10}$ Traducción del término oficial en inglés: Las "visions". 
ta, con la ampliación ya en un horizonte muy próximo, ha conocido la formulación y habilitación de programas y medios encaminados a facilitar el acercamiento de los países candidatos a unas condiciones político-institucionales, sociales y económicas mínimas que propicien su adaptación a la economía de mercado y el cumplimiento de requisitos marcados por la UE a todos ellos. El programa PHARE ha sido quizá el más extendido, consolidado y conocido. Programa de asistencia, ayuda y cooperación que gestiona la UE y que ha ido concediendo ayudas en forma de subvenciones y préstamos a proyectos (asistencia técnica, formación, estudios de viabilidad, reforma de las insituciones, reglamentación). El nombre es un acrónimo de "Poland, Hungary: Assistance or Restructuring Economies", aunque ya no son sólo Polonia y Hungría los países beneficiarios de este programa que entró en funcionamiento en 1990. Similar al PHARE pero extendido a los países de la antigua URSS es el programa TACIS (acrónimo de "Technical Assistance for the Commonwealth of Independent States"), que se puso en marcha en 1991. Para los Países del Este y Sur del Mediterráneo (los PESM) la UE configuró el Programa MEDA.

Los otros dos instrumentos económico-financieros específicamente creados para los países de la ampliación son el ISPA (Instruments for Structural Policies for Pre-accesion o Instrumento Estructural de Pre-Adhesión, con un sistema de funcionamiento similar al del Fondo de Cohesión de la propia UE, pues proyectos de protección del medio ambiente y de infraestructuras de transportes son las dos áreas prioritarias en que se centra su aplicación) y el SAPARD (Special Action Programme for Preaccesion Aid for Agriculture and Rural Development o Programa Especial de Adhesión para la Agricultura y el Desarrollo Rural). Su finalidad es la de ayudar a los países candidatos, previamente a la adhesión, a preparar su participación en la PAC y en el mercado interior, mediante medidas de ajuste estructural y de desarrollo rural.

\section{BIBLIOGRAFÍA}

AHIJAdo QuinTILlán, M. (2000): Historia de la unidad europea. Desde los precedentes remotos a la ampliación al Este; Madrid; Ed. Pirámide.

ANDERSON, M. \& BORT, E. (2000): The frontiers of the European Union; Hampshire; Palgrave; 235 pp.

Blas Guerrero, A. de (2003): «Quebec hoy, el País Vasco mañana»; Diario EL PAÍS, 4-V-2003; pg. 16.

CAMINAL BADÍA, M. (2003): «¿Quién teme a la federación?»; Diario EL PAÍS, 4-V-2003; pg. 16.

CAMPANELla, T. (1999): «Los desafíos de la UE en el umbral del siglo XXI»; en Politica Exterior, vol. XIII, n ${ }^{\circ}$ 69; pp. 41-57.

COMISIÓN EUROPEA (1995): Europa 2000+. Cooperación para la ordenación del territorio europeo; Bruselas-Luxemburgo. 
COMISIÓN EUROPEA (1999a): ETE. Estrategia Territorial Europea. Hacia un desarrollo equilibrado y sostenible del territorio de la UE; Oficina de $\mathrm{Pu}-$ blicaciones Oficiales de las Comunidades Europeas (OPOCE); Luxemburgo; $106 \mathrm{pp}$.

COMISIÓN EUROPEA (1999b): Sexto informe periódico sobre la situación y evolución socioeconómicas de las regiones de la Unión Europea; Oficina de Publicaciones Oficiales de las Comunidades Europeas (OPOCE); Luxemburgo; $242 \mathrm{pp}$.

COMISIÓN EUROPEA (2001a): Informe de la Comisión. Duodécimo Informe anual de los Fondos Estructurales (2000); COM(2001)539 final; Bruselas.

COMISIÓN EUROPEA (2001b): Unidad de Europa, Solidaridad de los pueblos, Diversidad de los territorios. Segundo Informe sobre la Cohesión Económica y Social; 2 vols.; 200 pp. Luxemburgo.

DELORS, J. (1995): "La personalidad política de Europa"; en Meridiano Ceri, $\mathrm{n}^{\circ}$ 4; pp. 4-9.

DOUCET, D. (2001): L'élargissement de l'Europe: un risque pour le Sud?; París; L'Harmattan; 283 pp.

EMERSON, M. (1999): El nuevo mapa de Europa; Alianza Editorial; Madrid.

ESTERUELAS, B. (2003): "El año de la refundación europea"; Diario EL PAÍS, 5-I-2003; pg. 6.

FLORES, G. Y LUENGO, F. (Coords.)(2000): Tras el muro: diez años después de 1989; Barcelona; El Viejo Topo.

FOUCHER, M. (Dir.)(1993): Fragments d'Europe; Fayard; París.

FOUCHER, M. (Dir.) ET AL. (1994): La próxima Europa. Un ensayo sobre alternativas y estrategias para una nueva visión de Europa; Fundación BBV; Bilbao.

FOUCHER, M. Y BAULAMON, C, (2002): «Europa Occidental y Central: una identidad en mutación»; en VV.AA.: El Estado del mundo 2003. Anuario económico geopolítico mundial; Madrid; Ed. Akal; pp. 418-421.

GARCÍA PICAZO, P. (1998): «Ideas en torno a la idea de Europa»; en Política y Sociedad, no 28; pp. 9-20.

LEPESANT, G. (2002): «Europa Occidental y Central: las tendencias del período»; en El Estado del mundo 2003. Anuario económico geopolítico mundial; Madrid; Ed. Akal; pp. 422-425.

LEVY, J. (1997): Europe. Une géographie; París; Hachette Supérieur; 288 pp. ORTEGA, A. (1999): «Un mapa de Europa»; Diario EL PAÍS; 18-X-1999; pg. 6

ORTEGA, A. (2000): «Una visión de la Europa del futuro»; Diario EL PAÍS; 18 XII-2000; pg. 18.

PECQUeRIE, B.; MunNICH, L. Y BASTENIER, M.A. (Coords.)(1993): Europa: el nuevo continente; Madrid; Diario EL PAÍS; Suplemento extraordinario; 80 pp. 
Petschen, S. (1998): «Una Europa de Estados, de Pueblos y de Regiones»; en Politica y Sociedad, $\mathrm{n}^{\mathrm{o}} 28$; pp.63-69.

Peyrony, J. Et. Hingray, M.C. (2002): Le schéma de développement de l'espace communautaire; París; La Documentation Française-Datar; 103 pp.

PLAZA GUTIÉRREZ, J.I. (1993): «El "factor regional” en el proceso de configuración territorial de la Europa contemporánea»; en Boletín de la Asociación de Geógrafos Españoles, $\mathrm{n}^{\circ}$ 17; Murcia; pp. 127-139.

PlAZA GuTIÉRREZ, J.I. (1997): «Europa en mutación (el Viejo Continente en el tránsito intersecular y ante un nuevo milenio)»; en CABERO DIÉGUEZ, V. Y PlAZA GUTIÉRREZ, J.I. (Coords.): Cambios regionales a finales del siglo $X X$; Asociación de Geógrafos Españoles (A.G.E.)-Departamento de Geografía de la Universidad de Salamanca; Salamanca; pp. 239-282.

PlAZA GUTIÉRREZ, J.I. (2000): «La política regional comunitaria: ¿una política solidaria?. Definición de sus objetivos e instrumentos de actuación. Perspectivas de futuro y valoración crítica»; en SAMANIEGO, M. Y GARCÍA, C. (Coords.): Europa solidaria. Actas del Seminario Intensivo. III Curso Superior de Historia de Integración de la Unión Europea; Cátedra Jean Monnet de Historia. Universidad de Salamanca; pp. 75-112.

PLAZA GUTIÉRREZ, J.I. (2002): «Orientaciones, conceptos e incertidumbres de la política regional europea para el siglo XXI»; Revista de Estudios Regionales, $\mathrm{n}^{\mathrm{o}}$ 64; pp. 43-74.

PLAZA GUTIÉRREZ, J.I. (2003a): «El territorio europeo ante el nuevo siglo»; Revista de Estudios Europeos, n 34; pp. 3-16.

PLAZA GUTIÉRREZ, J.I. (2003b): «El territorio europeo: grandes unidades, diferenciación de dinámicas internas, prácticas y políticas de intervención»; en SAMANIEGO, M. (Coord.): Reflexiones sobre Europa; Centro di Ricerca sull'integraziones europea. Università degli Studi di Siena. Dipartimento di Scienze Storiche, Giuridiche, Politiche e Sociali; Siena; pp. 81-98.

PlAZA GUTIÉRREZ, J.I. (2004): «Construcción o reinvención de Europa? Elementos de definición de una nueva geografía continental»; Cuadernos de Geografia, $\mathrm{n}^{\circ} 75$ (en prensa).

PRODI, R. (2000): Una idea de Europa; Madrid; Alianza Editorial; 190 pp.

Ruiz Molle, I. (1999): «La ordenación del territorio: una perspectiva europea»; en Revista de Economía y Finanzas de Castilla y León, $\mathrm{n}^{\circ} 2$; CajaDuero; pp. 73-93.

SOLÉ TURA, J. (2002): «¿Existe de verdad Europa?»; Diario EL PAÍS, 24-V2002; pg. 16.

TAIBO ARIAS, C. (1998): «Las otras Europas»; en Política y Sociedad, n 28; pp. 55-62. 
TAIBO ARIAS, C. (2000): ¿Un nuevo Tercer Mundo?. Una aproximación crítica a la Europa central y oriental contemporánea; A Coruña; Ed. Ludus; 145 pp.

TAIBO ARIAS, C. (2001): En el nuevo desorden. Capitalismo, conflictos e injerencia en la Europa central y oriental; Editorial Popular; 270 pp.

TOURAINE, A. (2003): «¿Existe Europa?»; Diario EL PAÍS, 20-I-2003; pp. 1112.

VICENTE I RUFÍ, J.V. (1993): «Construcción, deconstrucción y reconstrucción de Europa»; en Boletín de la Asociación de Geógrafos Españoles, $\mathrm{n}^{\circ}$ 17; Murcia; pp. 167-179.

VIDAL-FOLCH, X. (2002): «La segunda refundación de Europa, una oportunidad»; Diario EL PAÍS, 11-XII-2002; pp. 11-12.

RESUMEN: Después de establecer unas previas precisiones geográficas y de concepto, este artículo repasa algunos aspectos más destacados de lo que supone la quinta ampliación de la Unión Europea a través de algunos indicadores más representativos, se detiene en un breve perfil de los territorios que forman parte de este crecimiento espacial eurocomunitario y finaliza con una definición de los nuevos escenarios internacionales de integración continental que abre todo este proceso.

PALABRAS CLAVE: Ampliación; Europeidad; Recomposición; Mitteleuropa; Cooperación.

ABSTRACT: After establishing some previous geographical precisions and of concept, this article reviews some more outstanding aspects of what supposes the fifth amplification of the European Union through some more representative indicators, he/she stops in a brief profile of the territories that you/they are part of this growth space eurocomunitario and it concludes with a definition of the new international scenarios of continental integration that he/she opens this whole process.

KEY WORDS: Enlargement; European condition; Reconstruction; Mitteleuropa; Cooperation.

$\boldsymbol{R} \boldsymbol{E} \boldsymbol{S U} \boldsymbol{M} \boldsymbol{E}:$ Après quelques précisions géographiques et notionnelles préalables, le présent article passe en revue quelques-uns des aspects les plus marquants du cinquième élargissement de l'Union européenne par le biais des indicateurs les plus représentatifs, en décrivant brièvement par la suite le profil des territoires qui font partie de ce développement de l'espace eurocommunautaire, pour finaliser avec une définition de nouvelles scènes internationales d'intégration européenne surgies de ce processus.

MOTS-CLÉS: Élargissement ; européanité ; recomposition ; Mitteleuropa ; coopération

RESUMO: Depois de estabelecer umas prévias precisões geográficas e de conceito, este artigo repassa alguns dos aspectos mais destacados daquilo que supõe a quinta 
ampliação da União Europeia por meio de alguns dos indicadores mais representativos, detém-se num breve perfil dos territórios que tomam parte neste crescimento espacial eurocomunitário e conclui com uma definição dos novos cenários internacionais de integração continental que está a abrir todo este processo.

$\boldsymbol{P A L A V R A S ~ C H A V E : ~ A m p l i a c ̧ a ̃ o ; ~ E u r o p e i d a d e ; ~ R e c o m p o s i c ̧ a ̃ o ; ~ M i t t e l e u r o p a ; ~ C o o p e - ~}$ ração. 